\title{
A STUDY ON THE DETECTION OF BIOFILM FORMATION BY MULTIDRUG RESISTANT UROPATHOGENIC ISOLATES IN A TERTIARY CARE HOSPITAL
}

\author{
Samidurai Nalayini' ${ }^{1}$ Moongilpatti Ramasami Vasanthapriyan², Nagarajan Anuradha ${ }^{3}$, Daev Aravindh Moorthi ${ }^{4}$
}

${ }^{1}$ Assistant Professor, Institute of Microbiology, Madurai Medical College.

${ }^{2}$ Assistant Professor, Institute of Microbiology, Madurai Medical College.

${ }^{3}$ Assistant Professor, Institute of Microbiology, Madurai Medical College.

${ }^{4}$ MBBS Undergraduate Student (3rd Year).

\section{ABSTRACT}

\section{BACKGROUND}

Urinary Tract Infections (UTI) are the most common and important nosocomial infections, especially among diabetic patients. Emergence of multi-drug resistance and biofilm formation by these pathogens lead to chronic and recurrent infections. Our study aims at detection of multi-drug resistant uropathogens and biofilm formation by them.

Aims and Objective- Identification and isolation of significant multi-drug resistant uropathogens in diabetic patients. Detection of biofilm formation by these multi-drug resistant uropathogens by TCP, TM and CRA methods and comparison of these three methods for their efficacy.

Settings and Design- This is a hospital-based prospective cross-sectional study carried out among the Diabetic patients suffering from UTI in a tertiary care hospital for a period of six months.

\section{MATERIALS AND METHODS}

This study involved 127 Diabetic patients of both Type I and Type II, suffering from UTI. The urine samples collected were processed by standard microbiological techniques and the antibiotic susceptibility pattern of significant uropathogens were assessed. Uropathogens resistant to any three or more of the commonly used anti-microbial agents were considered multi-drug resistant and were assessed for biofilm formation by the following three methods- Tissue Culture Plate (TCP) method, Tube Method (TM) and Congo Red Agar (CRA) method using Pseudomonas aeruginosa ATCC 27853 strain and Staphylococcus epidermidis ATCC 12228 strains as controls. The results were analysed using the standard statistical methods.

\section{RESULTS}

In our study, the prevalence of UTI was higher in females (56.69\%) and in Type II Diabetic patients (91.34\%). Among the isolates, Escherichia coli was the commonest uropathogen. About 52\% of the isolates from urine samples were multi-drug resistant showing resistance to Penicillin, Cephalosporins and Sulphonamides. TCP method is found to be the standard method for the detection of biofilm formation. Biofilm formation and multi-drug resistance are found to be more in diabetic patients with poor glycaemic control.

\section{CONCLUSION}

Hence, this study reinforces the need for good glycaemic control in diabetic patients to prevent multi-drug resistance and biofilm formation by uropathogens.

\section{KEYWORDS}

UTI- Urinary Tract Infection, MDR- Multi-Drug Resistant, TCP- Tissue Culture Plate.

HOW TO CITE THIS ARTICLE: Nalayini S, Vasanthapriyan MR, Anuradha N, et al. A study on the detection of biofilm formation by multidrug resistant uropathogenic isolates in a tertiary care hospital. J. Evolution Med. Dent. Sci. 2017;6(80):5641-5646, DOI: $10.14260 /$ jemds/2017/1223

\section{BACKGROUND \\ Urinary Tract Infections (UTI) are the most common acquired bacterial infections and account for about $25 \%-40 \%$ of the nosocomial infections. ${ }^{[1]}$ Escherichia coli is the most common pathogen[2] followed by Enterococcus species, Staphylococcus saprophyticus, Proteus species, Klebsiella species, Pseudomonas aeruginosa and Candida albicans. Now-a-days,}

'Financial or Other Competing Interest': None.

Submission 16-08-2017, Peer Review 15-09-2017,

Acceptance 21-09-2017, Published 08-10-2017.

Corresponding Author:

Dr. Moongilpatti Ramasami Vasanthapriyan,

Assistant Professor,

Institute of Microbiology,

Madurai Medical College,

Madurai- 625020.

E-mail: drvasanth82@gmail.com

DOI: $10.14260 /$ jemds $/ 2017 / 1223$ emergence of multi-drug resistance among these pathogens is of serious concern and could be attributed due to inadequate dosage, improper and widespread use of broad spectrum antibiotics and transfer of anti-microbial resistant genes through plasmids. Biofilm formation by these pathogens worsens the situation further, as it protects them from opsonophagocytosis and antibiotics, thereby enhances its virulence leading to chronic, recurrent infections and sepsis.[3] Biofilms have medical significance, as they decrease their susceptibility to antimicrobial agents. ${ }^{[4]}$

More than $80 \%$ of all infections involve biofilms.[5] A biofilm is an assemblage of microbial cells, irreversibly associated with a surface and enclosed in a matrix of polysaccharide material.[6] The biofilm consists of layers of cell clusters embedded in a matrix of extracellular polysaccharide called polysaccharide intercellular adhesin (PIA), which consists of b-1, 6-N-acetylglycosamine and is synthesised by $\mathrm{N}$-acetylglucosamine transferase.[7] 
Biofilms are associated with indwelling medical devices like catheters, ventilators, implants and infections like dental caries, cystic fibrosis, osteonecrosis, urinary tract infections and eye infections. ${ }^{[8]}$

Both Gram positive and Gram negative bacteria like Enterococcus faecalis, Staphylococcus aureus, Staphylococcus epidermidis, Streptococcus viridans, Escherichia coli, Klebsiella pneumoniae, Proteus mirabilis and Pseudomonas aeruginosa ${ }^{[5]}$ are capable of biofilm formation.

Biofilm formation can be detected by Tissue Culture Plate Method (TCP), Tube Method (TM) and Congo Red Agar (CRA) Method.[5]

Microorganisms growing in a biofilm are intrinsically more resistant to antimicrobial agents than other organisms.[5] In the above perspective, this study proposes early detection of biofilm formation by the pathogens and their association with multi-drug resistance. Through this study, the three methods of biofilm detection are compared for their efficacy. This will guide the clinician for the institution of prompt and effective therapeutic measures for the infections, thereby reducing the morbidity and mortality associated with these infections.

\section{Aims and Objectives}

- To identify the significant pathogens with colony count of $\geq 10^{5} \mathrm{CFU} / \mathrm{mL}$, among the clinical isolates of Urinary Tract Infection in diabetic patients.

- To detect the multi-drug resistance among these significant uropathogens.

- To detect biofilm formation by TCP, TM and CRA methods.

- To compare the above methods in the detection of biofilm formation.

\section{MATERIALS AND METHODS}

\section{Study Centre and Duration}

This cross-sectional study was conducted in a tertiary care hospital for a duration of six months (August 2016 and January 2017) after obtaining Ethical Clearance from the Institutional Ethical Committee.

\section{Selection Criteria}

Diabetic patients presenting with the clinical features of urinary tract infections (UTI) like fever, urgency and frequency of urine, loin or abdominal pain and burning or painful micturition were included in the study.

Diabetic patients without the symptoms of UTI and the non-diabetic patients were excluded.

\section{Collection and Processing of Specimen}

Detailed clinical history of diabetic status of the patient was obtained. The patients were instructed to collect about 10 $20 \mathrm{~mL}$ of clean catch midstream urine into a wide-mouth screw cap sterile container after cleaning their external genitalia with soap and water. The urine samples were transported immediately to the Microbiology Laboratory for further processing.

The urine samples were processed by semi-quantitative standard calibrated loop method to assess the significant bacteriuria. The urine samples were processed further with Gram staining, motility by hanging drop method, solid media culture isolation, catalase test, oxidase test, coagulase test and other biochemical reactions. Antibiotic susceptibility pattern of these significant bacterial isolates were assessed by Kirby-Bauer disc diffusion method.

The pathogens resistant to any three or more of the following group of antimicrobials- Penicillins, Cephalosporins, Tetracyclines, Aminoglycosides, Macrolides, Quinolones and Sulfonamides were considered multi-drug resistant isolates.[9]

Biofilm production by these multi-drug resistant uropathogens were assessed by the following methodsTissue Culture Plate (TCP) method, Tube Method (TM) and Congo Red Agar (CRA) method. Pseudomonas aeruginosa ATCC 27853 and Staphylococcus epidermidis ATCC 12228 were used as controls.

\section{Tissue Culture Plate (TCA) Method}

The significant multi-drug resistant uropathogenic isolates were inoculated in $10 \mathrm{~mL}$ of trypticase soy broth with $1 \%$ glucose and incubated at $37^{\circ} \mathrm{C}$ for $24 \mathrm{hrs}$. Then they were diluted 1:100 using fresh trypticase soy broth. About $200 \mu \mathrm{L}$ of these individual cultures were loaded into the well of tissue culture plate along with the control strains and incubated at $37^{\circ} \mathrm{C}$ for $24 \mathrm{hrs}$. Sterile broth was included as a negative control. After incubation, the contents of the wells were discarded and wells were washed 4 times using $0.2 \mathrm{~mL}$ of phosphate-buffered saline. The biofilm formed by the pathogens adherent to the wells was fixed with $2 \%$ sodium acetate and then stained with $0.1 \%$ crystal violet. Then the wells were washed with deionised water and allowed to dry. The optical density of the stained biofilm was measured by ELISA reader at $492 \mathrm{~nm}$ wavelength.[5] The results were interpreted as non/weak, moderate and strong based on the criteria of Stepanovic et al[5,7] as mentioned below:-

\section{Interpretation of Biofilm Production by Tissue Culture} Plate (TCP) Method

\begin{tabular}{|c|c|}
\hline Average OD Value & Biofilm Production \\
\hline$\leq 2 \mathrm{x}$ ODc & Non/Weak \\
\hline$>2 \mathrm{x}$ ODc $\leq 4 \mathrm{x}$ ODc & Moderate \\
\hline$>4 \mathrm{x}$ ODc & Strong \\
\hline
\end{tabular}

Optical density cut-off value (ODc) = average OD of negative control $+3 x$ standard deviation (SD) of negative control.

\section{Tube Method (TM)}

Tube Method was performed by inoculating a loopful of the multi-drug resistant isolates in $10 \mathrm{~mL}$ of trypticase soy broth with $1 \%$ glucose in test tubes. They were then incubated at $37^{\circ} \mathrm{C}$ for 24 hrs. After incubation, the contents were discarded and the tubes were washed with phosphate buffer saline and dried. Then they were stained with $0.1 \%$ crystal violet and washed with deionised water and dried in inverted position. ${ }^{[5]}$ Observation of a visible film lining the wall of the tube was regarded as positive for biofilm formation.

\section{Congo Red Agar (CRA) Method}

The test isolates along with controls were inoculated onto the Congo red agar medium (Brain heart infusion broth $37 \mathrm{~g} / \mathrm{dL}$, Sucrose 50 g/dL, Agar No. $110 \mathrm{~g} / \mathrm{dL}$ and Congo Red Indicator $8 \mathrm{~g} / \mathrm{dL}$ ) the plates were then incubated aerobically at $37^{\circ} \mathrm{C}$ for 24 hrs.[5] After incubation, isolates showing black crystalline colonies were regarded as biofilm producers. 
The results were analysed by using the standard statistical methods.

\section{RESULTS}

A total of 127 samples were collected from the diabetic patients suffering from UTI. Out of 127 patients under study, 55 (43.31\%) were male patients and 72 (56.69\%) were female patients.

\begin{tabular}{|c|c|c|}
\hline Total No. of Samples Collected & Male & Female \\
\hline 127 & $55(43.31 \%)$ & $72(56.69 \%)$ \\
\hline \multicolumn{2}{|c|}{ Table 1. Sex Distribution of Samples } \\
\hline \multicolumn{2}{|c}{}
\end{tabular}

Out of 127 urine samples, 100 (78.74\%) samples showed microbial growth and 27 (21.26\%) showed no growth. Out of 100 positive urine cultures, $92(92 \%)$ were of monomicrobial growth and $8(8 \%)$ were of polymicrobial growth.

\begin{tabular}{|c|c|c|c|}
\hline $\begin{array}{c}\text { Total No. of Samples } \\
\text { Collected }\end{array}$ & \multicolumn{2}{|c|}{ Growth in Culture } & No. Growth \\
\hline 127 & \multicolumn{2}{|c|}{$100(78.74 \%)$} & $27(21.26 \%)$ \\
\hline & $\begin{array}{c}\text { Mono- } \\
\text { microbial } \\
\text { growth 92 }\end{array}$ & $\begin{array}{c}\text { Poly- } \\
\text { microbial } \\
\text { growth } 8\end{array}$ & \\
& $(92 \%)$ & $(8 \%)$ & \\
\hline
\end{tabular}

A total of 108 uropathogens were isolated from 100 samples. Out of 108 uropathogens, only 67 (62.04\%) isolates showed significant count of $\geq 10^{5} \mathrm{CFU} / \mathrm{mL}$ and were processed further.

Out of 67 significant uropathogenic isolates, 47 isolates were Gram Negative Bacilli (29 were Escherichia coli, 11 were Klebsiella sp. (Klebsiella pneumoniae-6 and Klebsiella oxytoca5, 2 were Pseudomonas sp., 2 were Proteus sp. and 3 were Citrobacter sp.) and 20 were Gram positive cocci (7 were Staphylococcus aureus, 4 were Staphylococcus epidermidis, 2 were Staphylococcus saprophyticus and 7 were Enterococcus sp.).

\begin{tabular}{|c|c|c|}
\hline \multicolumn{3}{|c|}{ No. of Isolates showing Significant count 67 } \\
\hline Uropathogen & No. of Isolates & Percentage \\
\hline Escherichia coli & 29 & $43.28 \%$ \\
\hline Klebsiella $s p$ & 11 & $16.42 \%$ \\
\hline Pseudomonas $s p$ & 2 & $2.98 \%$ \\
\hline Proteus $s p$ & 2 & $2.98 \%$ \\
\hline Citrobacter $s p$ & 3 & $4.48 \%$ \\
\hline Staphylococcus aureus & 7 & $10.45 \%$ \\
\hline Staphylococcus epidermidis & 4 & $5.97 \%$ \\
\hline $\begin{array}{c}\text { Staphylococcus } \\
\text { saprophyticus }\end{array}$ & 2 & $2.98 \%$ \\
\hline Enterococcus $s p$ & 7 & $10.45 \%$ \\
\hline
\end{tabular}

\section{Table 3. Distribution of Significant Uropathogenic Isolates}

The antibiotic susceptibility pattern of these 67 significant uropathogenic isolates were as follows: around 27 (40.30\%) were resistant to Gentamicin, 53 (79.10\%) were resistant to Nalidixic acid, $52(77.61 \%)$ were resistant to Norfloxacin, $18(26.87 \%)$ were resistant to Amikacin, 14 (20.90\%) were resistant to Nitrofurantoin, 7 (10.45\%) were resistant to Cefuroxime, 20 (29.85\%) were resistant to Cloxacillin, 35 (52.24\%) were resistant to Cotrimoxazole.

\begin{tabular}{|c|c|c|}
\hline Antibiotic Used & $\begin{array}{c}\text { No. of Isolates } \\
\text { Resistant }\end{array}$ & Percentage \\
\hline Gentamicin & 27 & $(40.30 \%)$ \\
\hline Nalidixic acid & 53 & $(79.10 \%)$ \\
\hline Norfloxacin & 52 & $(77.61 \%)$ \\
\hline Amikacin & 18 & $(26.87 \%)$ \\
\hline Nitrofurantoin & 14 & $(20.90 \%)$ \\
\hline Cefuroxime & 7 & $(10.45 \%)$ \\
\hline Cloxacillin & 20 & $(29.85 \%)$ \\
\hline Cotrimoxazole & 35 & $(52.24 \%)$ \\
\hline
\end{tabular}

\section{Table 4. Distribution of Antibiotic Resistance in Isolates}

The antibiotic resistance pattern observed in Gram negative (GNB) isolates were,

\section{Escherichia coli $(n=29)$}

Nalidixic acid (93.10\%) > Norfloxacin (86.21\%) > Cotrimoxazole $(62.07 \%)>$ Gentamicin $(41.38 \%)>$ Amikacin $(17.24 \%)>$ Cefuroxime $(13.79 \%)>$ Nitrofurantoin $(3.45 \%)$.

Klebsiella Species ( $n=11, K$. pneumoniae- 6, K. oxytoca- 5) Nalidixic acid (63.64\%), Norfloxacin (63.64\%) > Gentamicin $(54.55 \%)>$ Nitrofurantoin $(45.45 \%)$, Cotrimoxazole $(45.45 \%)>$ Amikacin $(27.27 \%)>$ Cefuroxime $(9.09 \%)$.

Pseudomonas Aeruginosa ( $n=2)$ : - Nalidixic acid (100\%), Nitrofurantoin $(100 \%)>$ Cotrimoxazole $(50 \%)$, Norfloxacin $(50 \%)$ and all were susceptible to Cefuroxime, Gentamicin and Amikacin.

\section{Proteus mirabilis $(n=2)$}

Nalidixic acid (50\%), Norfloxacin (50\%), Gentamicin (50\%), Cotrimoxazole (50\%) and all were susceptible to Cefuroxime, Nitrofurantoin and Amikacin.

\section{Citrobacter Species $(n=3)$}

Nalidixic acid $(100 \%)>$ Norfloxacin $(66.67 \%)$, Nitrofurantoin (66.67\%) > Gentamicin (33.33\%), Cotrimoxazole (33.33\%) and all were susceptible to Cefuroxime.

\begin{tabular}{|c|c|c|c|c|c|}
\hline Antibiotics & Escherichia coli (n= 29) & Klebsiella sp. (n= 11) & Pseudomonas sp. (n= 2) & $\begin{array}{c}\text { Proteus sp. (n= } \\
\text { 2) }\end{array}$ & Citrobacter sp.(n= 3) \\
\hline Gentamicin & $41.38 \%$ & $54.55 \%$ & $0 \%$ & $50 \%$ & $33.33 \%$ \\
\hline Nalidixic acid & $93.10 \%$ & $63.64 \%$ & $100 \%$ & $50 \%$ & $100 \%$ \\
\hline Norfloxacin & $86.21 \%$ & $63.64 \%$ & $50 \%$ & $50 \%$ & $66.67 \%$ \\
\hline Amikacin & $17.24 \%$ & $27.27 \%$ & $0 \%$ & $0 \%$ & $0 \%$ \\
\hline Nitrofurantoin & $3.45 \%$ & $45.45 \%$ & $100 \%$ & $0 \%$ & $66.67 \%$ \\
\hline Cefuroxime & $13.79 \%$ & $9.09 \%$ & $0 \%$ & $0 \%$ & $0 \%$ \\
\hline Cloxacillin & $20.69 \%$ & $18.18 \%$ & $0 \%$ & $100 \%$ & $33.33 \%$ \\
\hline Cotrimoxazole & $62.07 \%$ & $45.45 \%$ & $50 \%$ & $50 \%$ & $33.33 \%$ \\
\hline \multicolumn{7}{|r|}{ Table 5. Antibiotic Resistance \% of Gram Negative Bacilli } \\
\hline
\end{tabular}

The antibiotic resistance pattern of Gram Positive Cocci (GPC) were, 
Staphylococcus aureus $(n=7)$

Nalidixic acid (85.71\%), Norfloxacin (85.71\%) > Cotrimoxazole $(71.43 \%)>$ Nitrofurantoin $(28.57 \%)>$ Cloxacillin (14.29\%), Gentamicin (14.29\%), Amikacin (14.29\%), Cefuroxime (14.29\%).

\section{Staphylococcus epidermidis $(n=4)$}

Norfloxacin (75\%), Amikacin (75\%) > Cloxacillin (25\%), Cotrimoxazole (25\%), Cefuroxime (25\%) and all were susceptible to Nitrofurantoin and Gentamicin.

\section{Staphylococcus saprophyticus $(n=3)$}

Norfloxacin (100\%) > Cloxacillin (50\%), Cotrimoxazole (50\%), Cefuroxime (50\%) and all were susceptible to Nitrofurantoin and Gentamicin.

\section{Enterococcus sp. $(n=7)$}

High level Gentamicin (85.71\%) > Nalidixic acid (71.43\%), Norfloxacin (71.43\%), Amikacin (71.43\%) > Nitrofurantoin (42.86\%).

\begin{tabular}{|c|c|c|c|c|}
\hline Antibiotics & $\begin{array}{c}\text { Staphylococcus aureus } \\
(\mathbf{n = 7})\end{array}$ & $\begin{array}{c}\text { Staphylococcus epidermidis } \\
(\mathbf{n = 4 )}\end{array}$ & $\begin{array}{c}\text { Staphylococcus } \\
\text { saprophyticus } \\
(\mathbf{n}=\mathbf{2})\end{array}$ & $\begin{array}{c}\text { Enterococcus sp. } \\
(\mathbf{n}=7)\end{array}$ \\
\hline Gentamicin & $14.29 \%$ & $0 \%$ & $0 \%$ & $85.71 \%$ \\
\hline Nalidixic acid & $85.71 \%$ & $50 \%$ & $50 \%$ & $71.43 \%$ \\
\hline Norfloxacin & $85.71 \%$ & $75 \%$ & $100 \%$ & $71.43 \%$ \\
\hline Amikacin & $14.29 \%$ & $75 \%$ & $50 \%$ & $71.43 \%$ \\
\hline Nitrofurantoin & $28.57 \%$ & $0 \%$ & $0 \%$ & $42.86 \%$ \\
\hline Cefuroxime & $14.29 \%$ & $25 \%$ & $50 \%$ & - \\
\hline Cloxacillin & $14.29 \%$ & $25 \%$ & $50 \%$ & $42.86 \%$ \\
\hline Cotrimoxazole & $71.43 \%$ & $50 \%$ & $42.86 \%$ \\
\hline \multicolumn{7}{|r|}{ Table 6. Antibiotic Resistance \% of Gram Positive Cocci } \\
\hline
\end{tabular}

Out of 67 uropathogenic isolates, 35 were found to be resistant to Penicillins, Cephalosporins, Sulphonamides, Aminoglycosides and Fluoroquinolones; were identified as multi-drug resistant isolates (MDR). These isolates were subjected to Biofilm detection.

\section{Comparison of Detection Methods of Biofilm Formation} Out of 35 MDR isolates, 16 (45.71\%) were found to produce biofilm by Tissue Culture Plate (TCP) method, 15 (42.86\%) by Tube Method (TM) and 15 (42.86\%) by Congo Red Agar (CRA) method.

\begin{tabular}{|c|c|c|c|}
\hline $\begin{array}{c}\text { Detection } \\
\text { Method }\end{array}$ & $\begin{array}{c}\text { Total MDR } \\
\text { Isolate }\end{array}$ & $\begin{array}{c}\text { Biofilm } \\
\text { Producers }\end{array}$ & Percentage \\
\hline $\begin{array}{c}\text { Tissue Culture } \\
\text { Plate }\end{array}$ & 35 & 16 & $45.71 \%$ \\
\hline Tube method & 35 & 15 & $42.86 \%$ \\
\hline Congo red agar & 35 & 15 & $42.86 \%$ \\
\hline Table 7. Comparison of Biofilm Production by Various \\
Methods \\
\hline
\end{tabular}

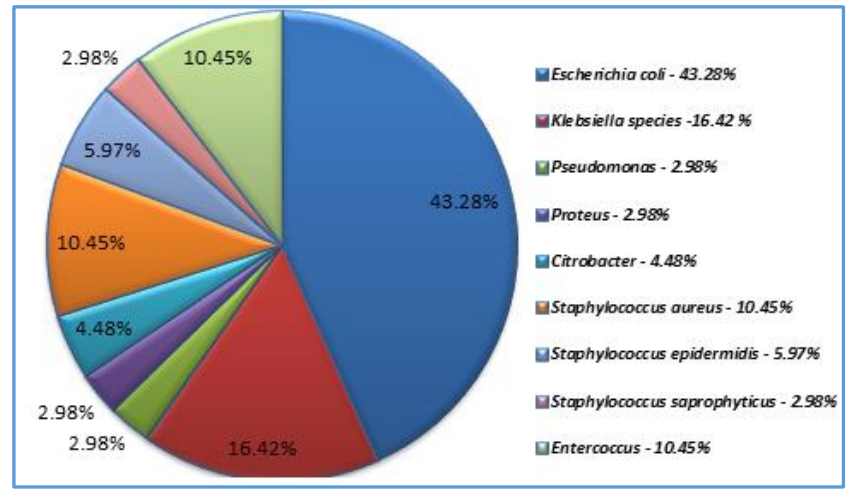

Chart 1. Distribution of Significant Uropathogenic Isolates

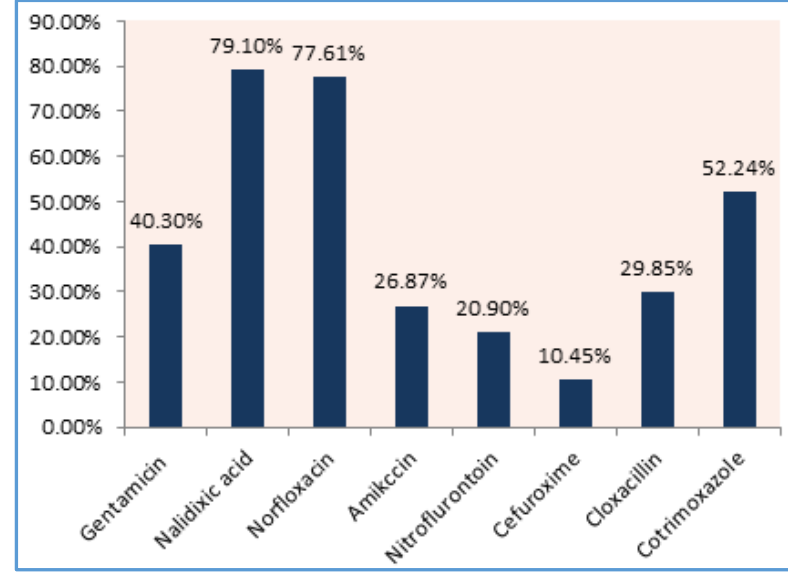

Chart 2. Overall Antibiotic Resistance Pattern

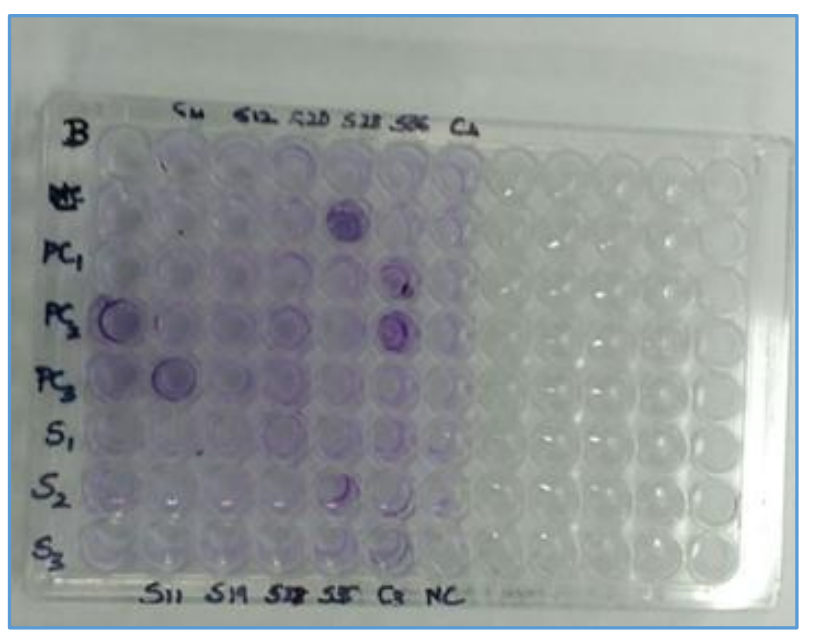

Figure 1. Biofilm Formation by TCP Method 


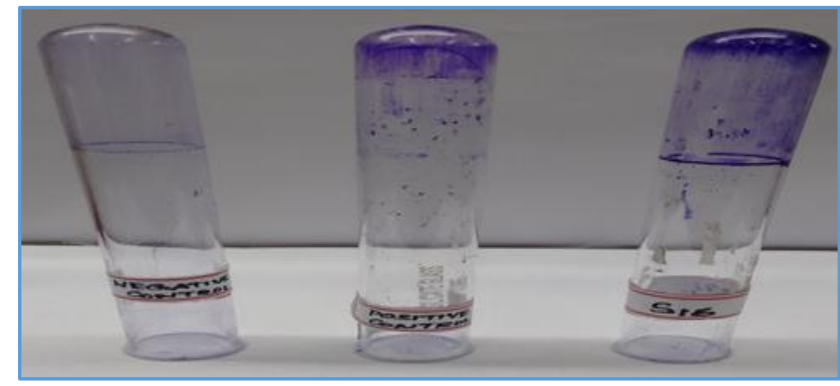

Figure 2. Biofilm Formation by Tube Method

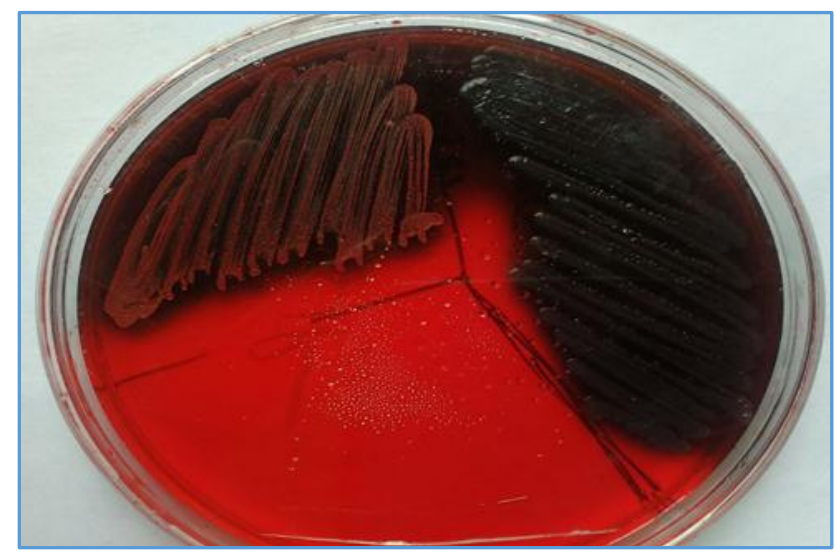

Figure 3. Congo Red Agar Method

\section{DISCUSSION}

In this study, we found that the prevalence of UTI were more in females $(56.69 \%)$ when compared to males $(43.31 \%)$. This was correlated with the finding of Getenet Beyene et al,[2] which tells that females $(64.9 \%)$ have high risk of UTI than males (35.1\%). It also correlates with the study by Arul Prakasam KC et al[10] and Devanand Prakash et al.[11] This strengthens the view that UTI infections are more common among female population.

In this study, UTI were common among type II diabetic patients (91.34\%) than type I diabetic patients (8.66\%). It correlates with the study done by Ramanath Katta Venkatesh et al,[12] which tells that $43 \%$ of diabetic patients have UTI. It also correlates with the study of Hamdan Z Hamdan et al[13] and S Niveditha et al.[1]

In our study, the polymicrobial pattern of growth was found to be $8 \%$. It was around $2.9 \%$ in the study done by J. Janifer et al.[14]

In this study Gram negative bacilli (73.15\%) were more common than Gram positive cocci $(26.25 \%)$, which correlates with the finding of Devanand Prakash et al[11] that out of 155 bacterial uropathogens, 140 (90.32\%) were Gram negative and 15 (9.68\%) were Gram positive isolates.

In this study, Escherichia coli (47.22\%) was the commonest isolate among the uropathogens. This coincides with the study done by Subramanian Pramodhini et al,[4] where E. coli was found to be the most frequently isolated uropathogen (70\%) and S Nivedhitha et al[1] study also signifies E. coli as the most frequently isolated organism.

In this study, the antimicrobial resistance pattern observed was Nalidixic acid (79.10\%) > Norfloxacin $(77.61 \%)>$ Cotrimoxazole $(52.24 \%)>$ Gentamicin $(40.30 \%)$ $>$ Amikacin (26.87\%) > Nitrofurantoin (20.90\%). This is similar to the pattern observed by Subramanian Pramodhini et al,[4] which reads as follows:- Nalidixic acid (84\%) > Norfloxacin $(72 \%)>$ Cotrimoxazole $(72 \%)>$ Gentamicin $(40 \%)>$ Nitrofurantoin (40\%) > Amikacin (20\%).

In this study, Escherichia coli $(28.57 \%)$ and Enterococcus sp. $(28.57 \%)$ were found to be most common biofilm producing organisms. It coincides with the study of Subramanian Pramodhini et al,[4] which shows $63 \%$ were Escherichia coli.

In this study, out of the 35 MDR isolates 16 (45.71\%) were biofilm producers by Tissue Culture Plate (TCP) method, 15 (42.86\%) by Tube Method (TM) and 15 (42.86\%) by Congo Red Agar (CRA) method. This correlates with the findings by Munesh Kumar Gupta et al.[15]

In this study, we found that the rate of biofilm formation was higher in the patients with poor glycaemic control compared to those with good glycaemic control correlating with the finding of May Sewify et al.[16]

\section{CONCLUSION}

The current study concludes that UTI is more common in female diabetic patients than male diabetic patients. Escherichia coli is the most common uropathogenic isolate. The uropathogens were more resistant to Nalidixic acid, Norfloxacin and fluoroquinolones and many are found to be multi-drug resistant isolates. Escherichia coli and Enterococcus $s p$. are the most common biofilm forming multidrug resistant uropathogens. Tissue culture plate method is found to be the standard method for the detection of biofilm formation. Biofilm formation and multi-drug resistance are found to be more in diabetic patients with poor glycaemic control. Hence, this study reinforces the need for good glycaemic control in diabetic patients in preventing emergence of multi-drug resistance and biofilm formation by uropathogens. It also emphasises initiation of early and appropriate anti-microbial therapy to reduce the mortality and morbidity associated with UTI in diabetic patients.

\section{REFERENCES}

[1] Niveditha S, Pramodhini S, Umadevi S, et al. The isolation and the biofilm formation of uropathogens in the patients with catheter associated urinary tract infections (UTIs). J Clin Diagn Res 2012;6(9):1478-82.

[2] Beyene G, Tsegaye W. Bacterial uropathogens in urinary tract infection and antibiotic susceptibility pattern in jimma university specialized hospital, southwest ethiopia. Ethiop J Health Sci 2011;21(2):141-6.

[3] Lotfi G, Hafida H, Nihel K, et al. Detection of biofilm formation by a collection of fifty strains of staphylococcus aureus isolated in Algeria at the university hospital of Tlemcen. Journal of Bacteriology Research 2014;6(1):1-6.

[4] Pramodhini S, Shanmugam N, Sivaraman U, et al. Antibiotic resistance pattern of biofilm-forming uropathogens isolated from catheterised patients in Pondicherry, India. Australas Med J 2012;5(7):344-8.

[5] Hassan A, Usman J, Kaleem F, et al. Evaluation of different detection methods of biofilm formation in the clinical isolates. Braz J Infect Dis 2011;15(4):30511. 
[6] Donlan RM. Biofilms: microbial life on surfaces. Emerg Infect Dis 2002;8(9):881-90.

[7] Oliveira A, Cunha Mde L. Comparison of methods for the detection of biofilm production in coagulasenegative staphylococci. BMC Res Notes 2010;3:260.

[8] Sawhney R, Berry V. Bacterial biofilm formation, pathogenicity diagnostics and control: an overview. Indian J Med Sci 2009;63(7):313-21.

[9] Magiorakos AP, Srinivasan A, Carey RB, et al. Multidrug-resistant, extensively drug-resistant and pandrug-resistant bacteria: an international expert proposal for interim standard definitions for acquired resistance. Clin Microbiol Infect 2012;18(3):268-81.

[10] Prakasam AKC, Kumar DKG, Vijayan M. A cross sectional study on urinary tract infection and their antibiotic utilisation pattern in Kerala. Int J Pharm Tech Res 2012;4(3):1309-16.

[11] Prakash D, Saxena RS. Distribution and antimicrobial susceptility pattern of bacterial pathogens causing urinary tract infection in urban community of meerut city, India. ISRN Microbiology 2013;2013:pp 13.
[12] Venkatesh RK, Prabhu MM, Nandakumar K, et al. Urinary tract infection treatment pattern of elderly patients in a tertiary hospital setup in south India: a prospective study. J Young Pharm 2016;8(2):108-13.

[13] Hamdan HZ, Kubbara E, Adam AM, et al. Urinary tract infections and antimicrobial sensitivity among diabetic patients at Khartoum, Sudan. Ann Clin Microbiol Antimicrob 2015;14:26.

[14] Janifer J, Geethalakshmi S, Satyavani K, et al. Prevalence of lower urinary tract infection in South Indian type 2 diabetic subjects. Indian J Nephrol 2009;19(3):107-11.

[15] Gupta MK, Gahlot R, Nigam C, et al. Biofilm: detection methods and correlation with antimicrobial resistance in staphylococcus. National Journal of Laboratory Medicine 2013;2(2):7-10.

[16] Sewify M, Nair S, Warsame S, et al. Prevalance of urinary tract infections and antiicrobial suscepitibility among diabetic patients with controlled and uncontrolled glycemia in Kuwait. Journal of Diabetes Research 2016;2016:pp 7. 